\title{
Controlling Catalyst Bulk Reservoir Effects for Monolayer Hexagonal Boron Nitride CVD
}

Sabina Caneva, ${ }^{\dagger}$ Robert S. Weatherup, ${ }^{\dagger \neq}$ Bernhard C. Bayer, ${ }^{\dagger, \S}$ Raoul Blume, ${ }^{\|}$Andrea Cabrero-Vilatela, ${ }^{\dagger}$ Philipp Braeuninger-Weimer, ${ }^{\dagger}$ Marie-Blandine Martin, ${ }^{\dagger}$ Ruizhi Wang, ${ }^{\dagger}$ Carsten Baehtz, ${ }^{\perp}$ Robert Schloegl, ${ }^{\nabla}$ Jannik C. Meyer, ${ }^{\ddagger}$ and Stephan Hofmann*, ${ }^{\dagger}$

${ }^{\dagger}$ Department of Engineering, University of Cambridge, JJ Thomson Avenue, CB3 OFA Cambridge, United Kingdom

${ }^{\ddagger}$ Materials Sciences Division, Lawrence Berkeley National Laboratory, 1 Cyclotron Road, Berkeley, California 94720, United States

${ }^{\S}$ Faculty of Physics, University of Vienna, Boltzmanngasse 5, A-1090 Vienna, Austria

"Helmholtz-Zentrum Berlin für Materialen und Energie, D-12489 Berlin, Germany

${ }^{\perp}$ Institute of Radiation Physics, Helmholtz-Zentrum Dresden-Rossendorf, 01314 Dresden, Germany

$\nabla$ Fritz Haber Institute, D-14195 Berlin-Dahlem, Germany

Supporting Information

ABSTRACT: Highly controlled Fe-catalyzed growth of monolayer hexagonal boron nitride (h-BN) films is demonstrated by the dissolution of nitrogen into the catalyst bulk via $\mathrm{NH}_{3}$ exposure prior to the actual growth step. This "pre-filling" of the catalyst bulk reservoir allows us to control and limit the uptake of $\mathrm{B}$ and $\mathrm{N}$ species during borazine exposure and thereby to control the incubation time and h-BN growth kinetics while also limiting the contribution of uncontrolled precipitation-driven h-BN growth during cooling. Using in situ X-ray diffraction and in situ X-ray photoelectron spectroscopy combined with systematic growth calibrations, we develop an understanding and framework for engineering the catalyst bulk reservoir to optimize the growth process, which is also relevant to other $2 \mathrm{D}$ materials and their heterostructures.

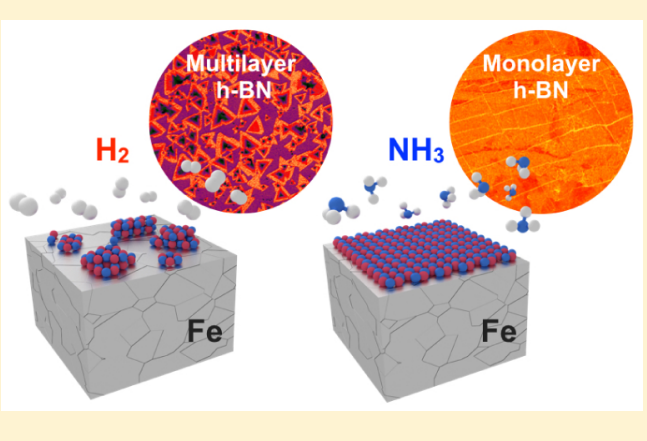

KEYWORDS: hexagonal boron nitride $(h-B N)$, chemical vapor deposition $(\mathrm{CVD})$, borazine $(\mathrm{HBNH})_{3}$, ammonia $\left(\mathrm{NH}_{3}\right)$, iron $(\mathrm{Fe})$

$\mathrm{H}$ exagonal boron nitride $(\mathrm{h}-\mathrm{BN})$ is a two-dimensional (2D) dielectric material isostructural to graphene whose distinctive properties make it highly promising as a barrier-, spacer-, or support-layer for future integrated electronics and photonics. $^{1-3}$ A key technological challenge, as for all $2 \mathrm{D}$ materials, is the scalable manufacture of $\mathrm{h}-\mathrm{BN}$, in particular as a continuous film of controlled layer number, crystalline quality, and microstructure. ${ }^{4-6}$ Chemical vapor deposition (CVD) has emerged as the most viable technique to address this, as it enables not only bulk production but importantly also controlled interfacing and direct material integration; that is, it enables integrated manufacturing. ${ }^{7}$ Critical to the h-BN CVD process is the use of a catalyst that, similar to graphene CVD, facilitates low activation energy pathways for precursor dissociation, h-BN nucleation, domain growth, and merging. ${ }^{8-10}$ Mono- and few-layer h-BN CVD has been reported on a range of transition metal catalysts; ${ }^{11-25}$ however, the growth mechanisms under scalable conditions remain unclear, thus limiting control over h-BN film structure as well as the more complex direct CVD of $2 \mathrm{D}$ heterostructures. ${ }^{26-28}$ The optimization of the CVD process is typically focused on preparation of the catalyst surface prior to the growth, for example, by mechanical or chemical polishing, ${ }^{11,29}$ and for the given choice of precursor and its dilution, the temperature, pressure, and time of the actual CVD growth step. The role of the reaction atmosphere during preannealing has not been discussed in great detail in literature for 2D h-BN layers, although it has been investigated for carbon and boron nitride nanotubes, as well as for graphene CVD. ${ }^{30-33}$

It has been shown that the boron (B) and nitrogen (N) solubility and permeability are strongly catalyst dependent; for instance, for common catalysts such as $\mathrm{Fe}$ or $\mathrm{Cu}$, both species dissolve in $\mathrm{Fe}$, but only $\mathrm{B}$ dissolves in $\mathrm{Cu}$ to any significant level. ${ }^{34,35}$ Typically, such non-negligible solubilities are considered deleterious and have not been considered as parameters to enhance growth control. ${ }^{36}$ The finite concentration of constituent elements that can be dissolved into the catalyst bulk has two key implications: (i) it can affect the catalyst phase evolution during CVD and (ii) the resulting bulk reservoir effect can alter the growth kinetics. ${ }^{37}$

Here, we show that the dissolution of species into the catalyst bulk prior to the actual growth step can be used to significantly

Received: November 10, 2015

Revised: January 8, 2016

Published: January 12, 2016 
improve the homogeneity and growth control for h-BN CVD. We focus on Fe-catalyzed h-BN CVD with borazine and show that $\mathrm{Fe}$ exposure to $\mathrm{NH}_{3}$ before the actual growth step leads to the dissolution of $\mathrm{N}$ species into the Fe bulk. This "pre-filling" of the catalyst bulk reservoir allows us to control and limit the uptake of $\mathrm{B}$ and $\mathrm{N}$ species during borazine exposure and thereby limit the contribution of precipitation-driven growth. It also enables us to control the incubation time and growth kinetics, through which we achieve exclusive growth of uniform monolayer h-BN films on $\mathrm{Fe}$ foils. Using in situ X-ray diffraction (XRD) and in situ X-ray photoelectron spectroscopy (XPS) combined with a systematic growth study and ex situ characterization, we develop an in-depth understanding of the growth kinetics and possibilities for engineering the catalyst bulk reservoir to optimize the CVD process. Our insights are relevant to all catalyst materials and the kinetic growth model offers a general framework relevant also to other $2 \mathrm{D}$ materials and their heterostructures.

Results. We adopt a simple CVD process, the salient stages of which are outlined in Figure 1: (1) the preannealing stage,

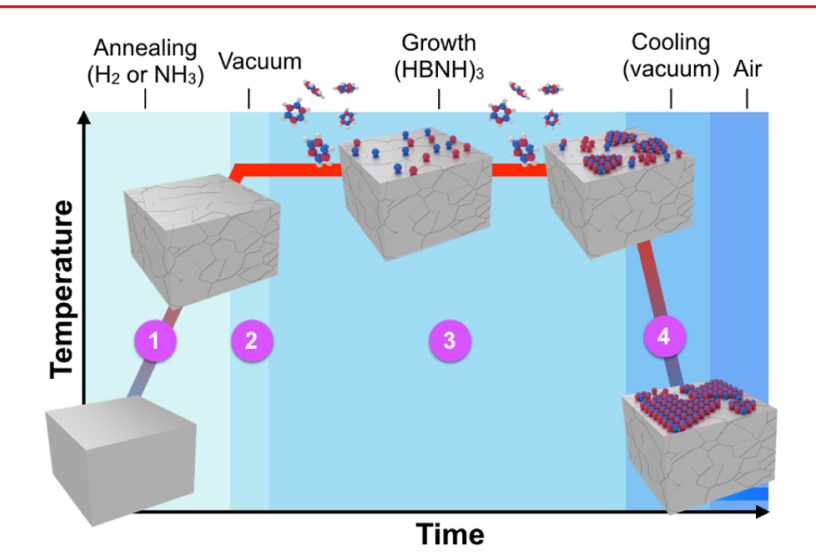

Figure 1. Schematic of the salient stages of the CVD process. The shaded areas correspond to the preannealing stage in either $\mathrm{H}_{2}$ or $\mathrm{NH}_{3}$ (1), the vacuum stage prior to borazine exposure (2), the borazine exposure period (3), and the vacuum cooling stage (4). during which the samples are gradually heated in $\mathrm{H}_{2}$ or $\mathrm{NH}_{3}$, (2) the vacuum stage preceding borazine exposure, (3) the borazine exposure period, and (4) the vacuum cooling stage. Figure 2 compares the morphology of the h-BN grown on asreceived $\mathrm{Fe}$ foils $(100 \mu \mathrm{m})$ at $\sim 900{ }^{\circ} \mathrm{C}$ and $6 \times 10^{-4} \mathrm{mbar}$ borazine partial pressure following preannealing in 4 mbar of either $\mathrm{H}_{2}$ or $\mathrm{NH}_{3}$ (Stage 1) as a function of borazine exposure time (Stage 3). We observe a strong effect of the preannealing gas on the growth. In particular, for the $\mathrm{NH}_{3}$ preannealing, an increase in growth time results in a gradual closure of the monolayer h-BN film, without multilayer formation. Conversely, the $\mathrm{H}_{2}$ preannealing does not lead to a continuous, homogeneous h-BN film even for extended growth times, and primarily results in thicker, few-layer h-BN domains.

The average domain size for the $\mathrm{H}_{2}$-preannealed sample is $<1$ $\mu \mathrm{m}$ after $45 \mathrm{~s}$ borazine exposure (Figure 2a). For a $14 \mathrm{~s}$ borazine exposure following a $\mathrm{H}_{2}$ preannealing (Figure 2a inset) no h-BN was observed on the Fe surface, indicative of an incubation period preceding crystal nucleation of at least $14 \mathrm{~s}$. We note that the h-BN domain orientation, size, and density varies across the sample surface, suggesting $\mathrm{Fe}$ grain orientation-dependent growth kinetics, a feature that is also observed for the $\mathrm{NH}_{3}$-preannealed foil (see Supporting Information Figure S1). After $90 \mathrm{~s}$, the domains grow to average lateral sizes of $\sim 3 \mu \mathrm{m}$ (Figure $2 \mathrm{~b}$ ), and the change in contrast from the edges to the center of the triangular domains also indicates a notable thickness variation, with brighter regions corresponding to multilayers and conversely dark gray regions indicating monolayer h-BN. Extended growth times (480 s, Figure 2c) lead to slightly higher h-BN coverages; however, growth appears to primarily proceed through the formation of additional h-BN layers, rather than lateral expansion of the existing layers. Additionally, we observe a bimodal domain size distribution across the entire sample surface (Figure S2), with large domains (side length $\sim 15 \mu \mathrm{m}$ ) coexisting with domains about ten times smaller. This observation suggests that two distinct growth regimes appear to exist under these growth conditions for the $\mathrm{H}_{2}$ preannealing.

For the $\mathrm{NH}_{3}$-preannealed Fe foil, the most notable difference after $45 \mathrm{~s}$ borazine exposure is the formation of a lower density of triangular domains with lateral dimensions of $\sim 20 \mu \mathrm{m}$

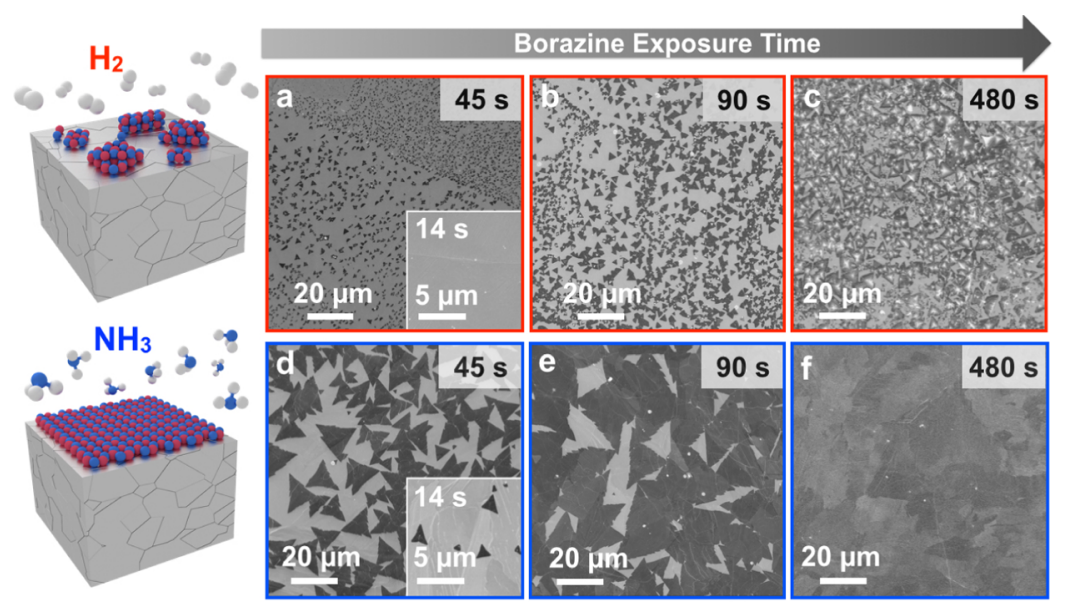

Figure 2. SEM images of h-BN domains grown at $\sim 900{ }^{\circ} \mathrm{C}$ and $6 \times 10^{-4}$ mbar borazine exposure on $(\mathrm{a}, \mathrm{b}, \mathrm{c}) \mathrm{H}_{2}$-preannealed $\mathrm{Fe}$ foil for $45 \mathrm{~s}, 90 \mathrm{~s}$, and $480 \mathrm{~s}$ borazine exposure time, respectively, and on $(\mathrm{d}, \mathrm{e}, \mathrm{f}) \mathrm{NH}_{3}$-preannealed Fe foil for $45 \mathrm{~s}, 90 \mathrm{~s}$, and $480 \mathrm{~s}$ borazine exposure time, respectively. The preannealing pressure in both $\mathrm{H}_{2}$ and $\mathrm{NH}_{3}$ is 4 mbar for all of the above growths. The insets in $(\mathrm{a}, \mathrm{d})$ correspond to $14 \mathrm{~s}$ exposures and illustrate the longer incubation time preceding nucleation of h-BN on the $\mathrm{H}_{2}$-preannealed sample. 

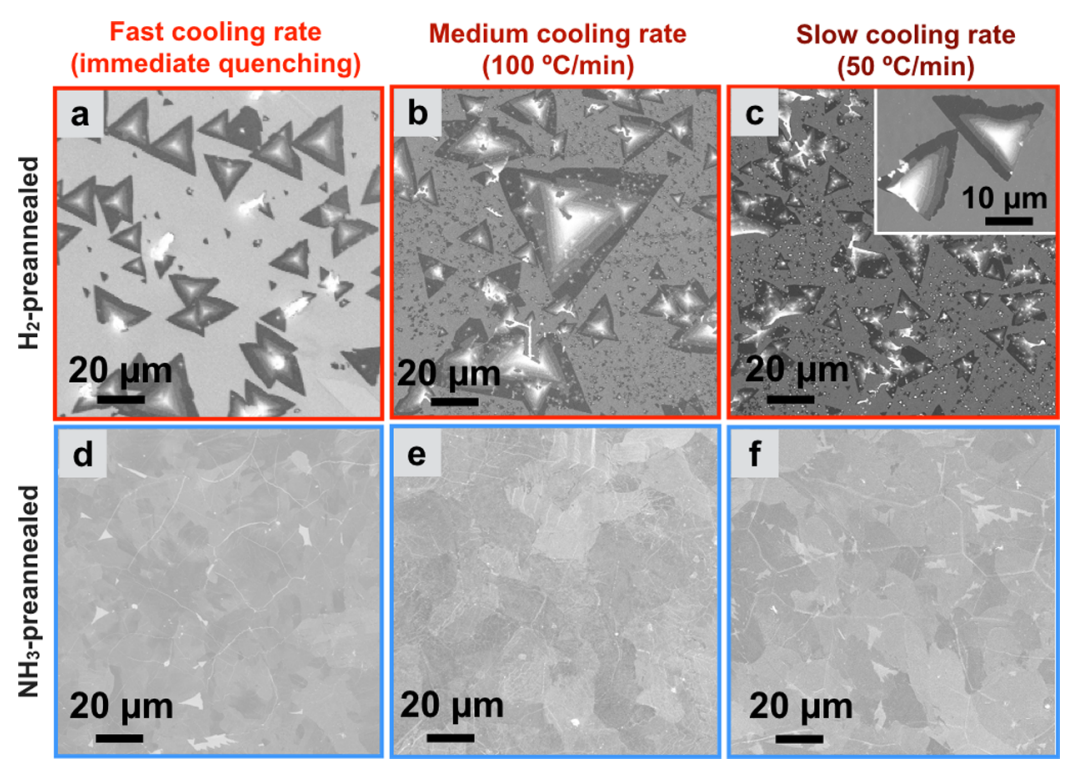

Figure 3. Morphology of h-BN on $\mathrm{H}_{2}$-preannealed Fe foil after (a) fast cooling $\left(300^{\circ} \mathrm{C}\right)$, (b) medium cooling $\left(100{ }^{\circ} \mathrm{C} / \mathrm{min}\right)$, and (c) slow cooling $\left(50^{\circ} \mathrm{C} / \mathrm{min}\right)$. Inset: detail of multilayered domains with irregular edges, indicating possible dissolution of material back into the bulk during slow cooling. Morphology of h-BN on $\mathrm{NH}_{3}$-preannealed $\mathrm{Fe}$ foil after (d) fast cooling, (e) medium cooling, and (f) slow cooling. All growths were performed at $\sim 900^{\circ} \mathrm{C}$ and $6 \times 10^{-4}$ mbar borazine exposure for $5 \mathrm{~min}$.
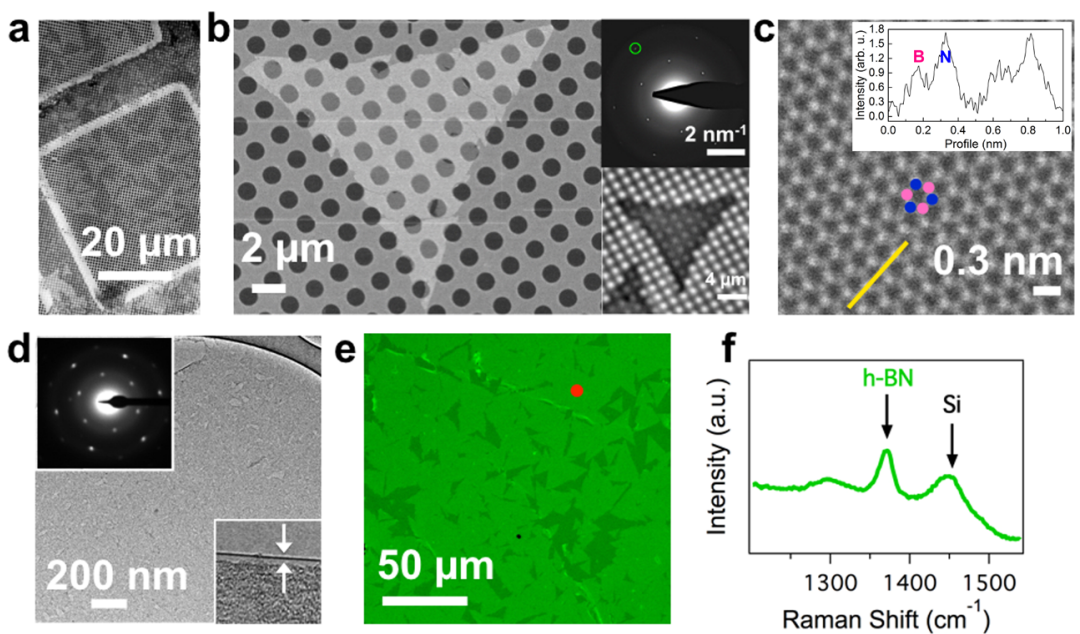

Figure 4. (a) SEM image of h-BN domains from Figure $2 \mathrm{~d}$ transferred onto a holey carbon/copper TEM grid. (b) DF-TEM image of a triangular h$\mathrm{BN}$ domain, confirming its single-crystalline nature. We note that the image is composed of several stitched individual frames. The upper right inset shows the corresponding hexagonal diffraction pattern with the diffraction spot, from which the DF-TEM image was acquired, indicated by the green circle. The lower right inset shows a defocused BF-TEM image demonstrating that the island is indeed isolated. (c) High-angle annular dark field (HAADF) STEM image from the center of a triangular domain showing atomic and element specific contrast. The image was processed following Krivanek et al. to reduce contributions from probe-tails. ${ }^{39}$ The intensity profile along the indicated yellow line (inset) exhibits an N/B intensity ratio consistent with monolayer h-BN. ${ }^{39}$ (d) Bright-field TEM image of a suspended h-BN film from the sample in Figure $2 \mathrm{f}$. The top left inset shows the diffraction pattern from this region and the bottom right inset is an edge-analysis, confirming the monolayer nature of the film. (e) Optical image after transfer of the sample in Figure 1e, showing coalescing h-BN domains. We note that the image was acquired using a green filter to enhance the contrast. (f) Raman spectrum acquired in the region indicated by the red dot in panel (e), displaying the characteristic h-BN peak at $1369 \mathrm{~cm}^{-1}$ and the Si-related peak. ${ }^{59}$

(Figure 2d), which are more than ten times the size of the domains grown on the $\mathrm{H}_{2}$-preannealed $\mathrm{Fe}$ foil for the same exposure time. We also note that the edges are sawtoothed, a feature that we have observed previously for large h-BN domains grown on $\mathrm{Fe} / \mathrm{SiO}_{2} / \mathrm{Si}$ substrates, ${ }^{34}$ and which can be ascribed to diffusion instabilities. ${ }^{38}$ The incubation time for the $\mathrm{NH}_{3}$-preannealed catalyst is shorter, highlighted by the appearance of triangular domains after just $14 \mathrm{~s}$ of borazine exposure (Figure 2d inset). The h-BN domains are largely merged after $90 \mathrm{~s}$ exposure (Figure 2e), with only a few gaps left in the film. After $480 \mathrm{~s}$ of borazine exposure, we achieve full coverage of the Fe catalyst with monolayer h-BN (Figure 2f). The remaining variations in SEM contrast over the surface of the sample are due to channeling contrast from the polycrystalline Fe foil.

Figure 3 summarizes the effect of three different cooling rates (Stage 4 in Figure 1) on the h-BN morphology after growth on the differently preannealed foils $\left(\mathrm{H}_{2}\right.$ or $\mathrm{NH}_{3}, 4$ mbar $)$, followed by a fixed borazine exposure $\left(6 \times 10^{-4} \mathrm{mbar}, \sim 900^{\circ} \mathrm{C}, 5 \mathrm{~min}\right)$. We note that the cooling rates refer to the initial cooling period 


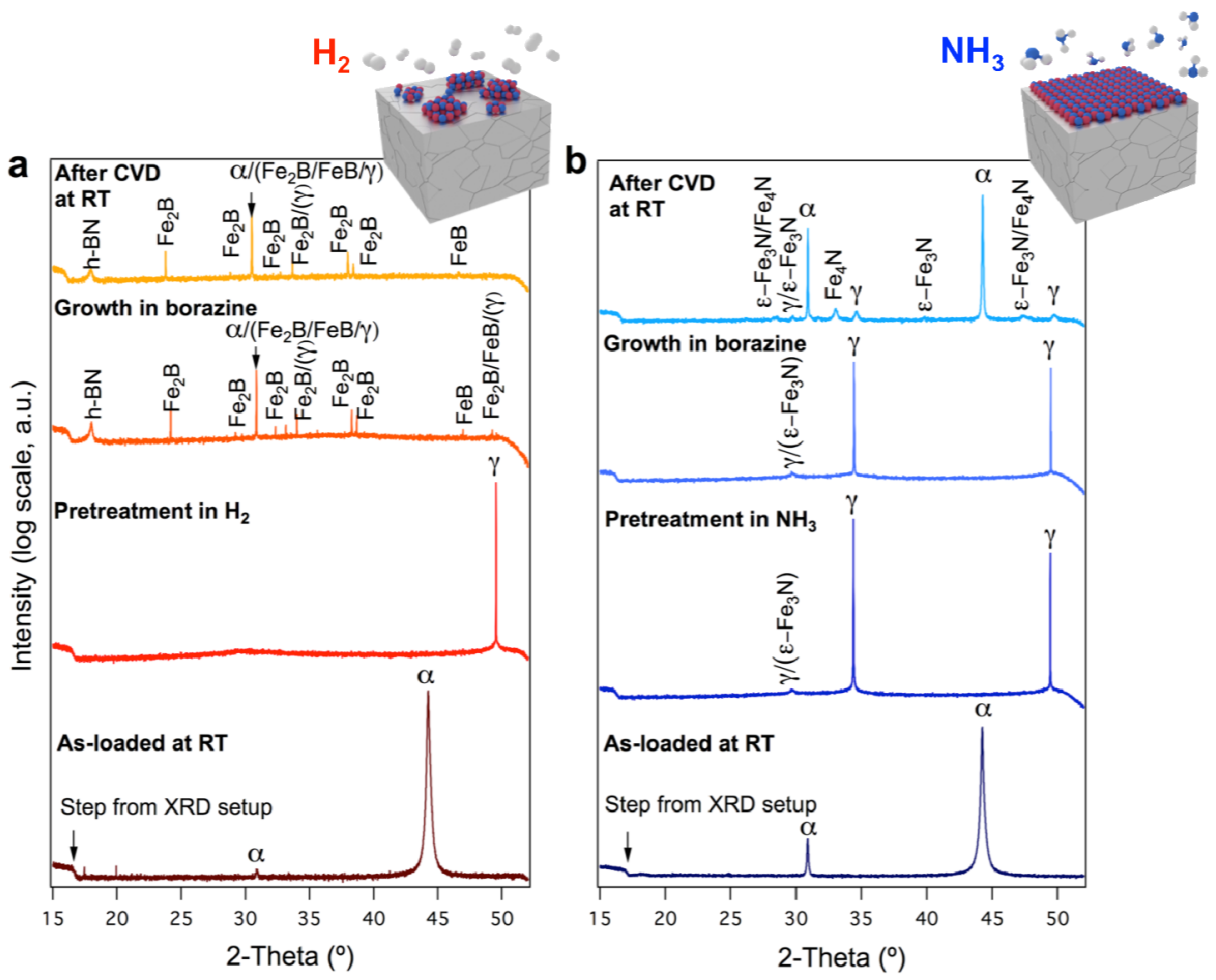

Figure 5. In situ XRD patterns of Fe foil catalyzed h-BN growth during the salient stages of the CVD process, comparing the $\mathrm{H}_{2}$ preannealing (a) and the $\mathrm{NH}_{3}$ preannealing (b). The labeling indicates possible phase assignments with less likely phase assignments in brackets. We note that intensity is plotted here on a log scale to emphasize minority phases. The catalyst was annealed in $2.6 \mathrm{mbar}^{\circ} \mathrm{H}_{2}$ (a) and $4 \mathrm{mbar}$ of $\mathrm{NH}_{3}$ (b), and the growth was performed at $\sim 900{ }^{\circ} \mathrm{C}$ (estimated uncertainty in temperature $\pm 25{ }^{\circ} \mathrm{C}$ ) in $\sim 6 \times 10^{-4}$ mbar borazine partial pressure for 10 min.

from $\sim 900{ }^{\circ} \mathrm{C}$ down to $\sim 500{ }^{\circ} \mathrm{C}$, after which the rate slows down and is comparable for all three cases. Figure $3 \mathrm{a}$ corresponds to immediate quenching $\left(\sim 300{ }^{\circ} \mathrm{C} / \mathrm{min}\right)$ for a $\mathrm{H}_{2}$-preannealed foil, with the heater turned off immediately after borazine exposure, and shows a bimodal domain size distribution of few-layer h-BN. For an intermediate cooling rate of $100{ }^{\circ} \mathrm{C} / \mathrm{min}$, the $\mathrm{h}-\mathrm{BN}$ domains appear larger and more multilayered, and the density of the smaller domains is considerably higher (Figure $3 \mathrm{~b}$ ), indicating that some portion of the h-BN is formed on cooling. For slow cooling rates of 50 ${ }^{\circ} \mathrm{C} / \mathrm{min}$, the domains are similar in size and thickness to the intermediate cooling rate; however, the shapes are no longer strictly triangular and the edges become less sharp (Figure 3c inset). The same cooling experiments were performed on the $\mathrm{NH}_{3}$-preannealed foils (Figure $3 \mathrm{~d}-\mathrm{f}$ ). The h-BN domains remain as monolayers, with no multilayers observed for any of the cooling rates used here. This illustrates that, contrary to the $\mathrm{H}_{2}$-preannealing case, additional layer formation and nucleation do not occur on cooling, both of which are effects that can be linked to precipitation of $\mathrm{B}$ and $\mathrm{N}$ species from the catalyst bulk.

To further understand this difference in behavior with preannealing atmosphere, we performed a growth where we first preannealed the sample in $\mathrm{NH}_{3}$, and then held it at the growth temperature in vacuum for 30 min after the $\mathrm{NH}_{3}$ preanneal but prior to borazine exposure (i.e., extended Stage 2 in Figure 1). Figure S3 shows the postgrowth surface of this sample, which consists of a h-BN domain size distribution tending to be more bimodal than for the standard growth with the $\mathrm{NH}_{3}$ preannealing. Furthermore, most of the domains are smaller and multilayered, and are reminiscent of those grown on the $\mathrm{H}_{2}$-preanneled sample, despite the lower nucleation density.

The structure and crystallinity of the h-BN formed by the optimized process using $\mathrm{NH}_{3}$ preanneling was determined by transmission electron microscopy (TEM)/scanning transmission electron microscopy (STEM). Figure 4a shows a scanning electron microscopy (SEM) image of a holey carbon/ copper TEM grid after transferring isolated h-BN domains corresponding to Figure $2 \mathrm{~d}$. Figure $4 \mathrm{~b}$ shows a dark-field TEM (DF-TEM) image of one such h-BN island, confirming the single-crystalline nature of the domain. The corresponding selected area electron diffraction pattern (upper right inset) shows one hexagonal set of diffraction spots also consistent with single-crystalline $\mathrm{h}$-BN. The green circle indicates the diffraction spot used to produce the DF-TEM image. The lower right inset shows a defocused bright-field (BF) TEM image confirming that the domain is indeed isolated, and a second differently oriented h-BN domain can be partially seen in the lower left corner. We further analyze the film by high angle annular dark field (HAADF) STEM, which reveals atomic and element specific contrast. ${ }^{39}$ The high-resolution STEM image in Figure $4 c$ displays the hexagonal lattice of h-BN and the intensity profile along the marked yellow line exhibits a clear distinction of the $\mathrm{B}$ and $\mathrm{N}$ atoms (Figure $4 \mathrm{c}$ inset), where the extracted intensity ratio is consistent with monolayer h-BN. ${ }^{39}$ Further confirmation of the monolayer nature of the h-BN was obtained by electron beam induced sputtering in Supporting Information Figure S4, which exhibits direct, step-free sputtering of the layer to vacuum consistent with single-layer h-BN (whereas multilayer h-BN is sputtered in a layer-by-layer fashion). ${ }^{40-42}$ Figure $4 \mathrm{~d}$ shows a bright-field TEM image of a suspended closed h-BN film, with the corresponding diffraction 

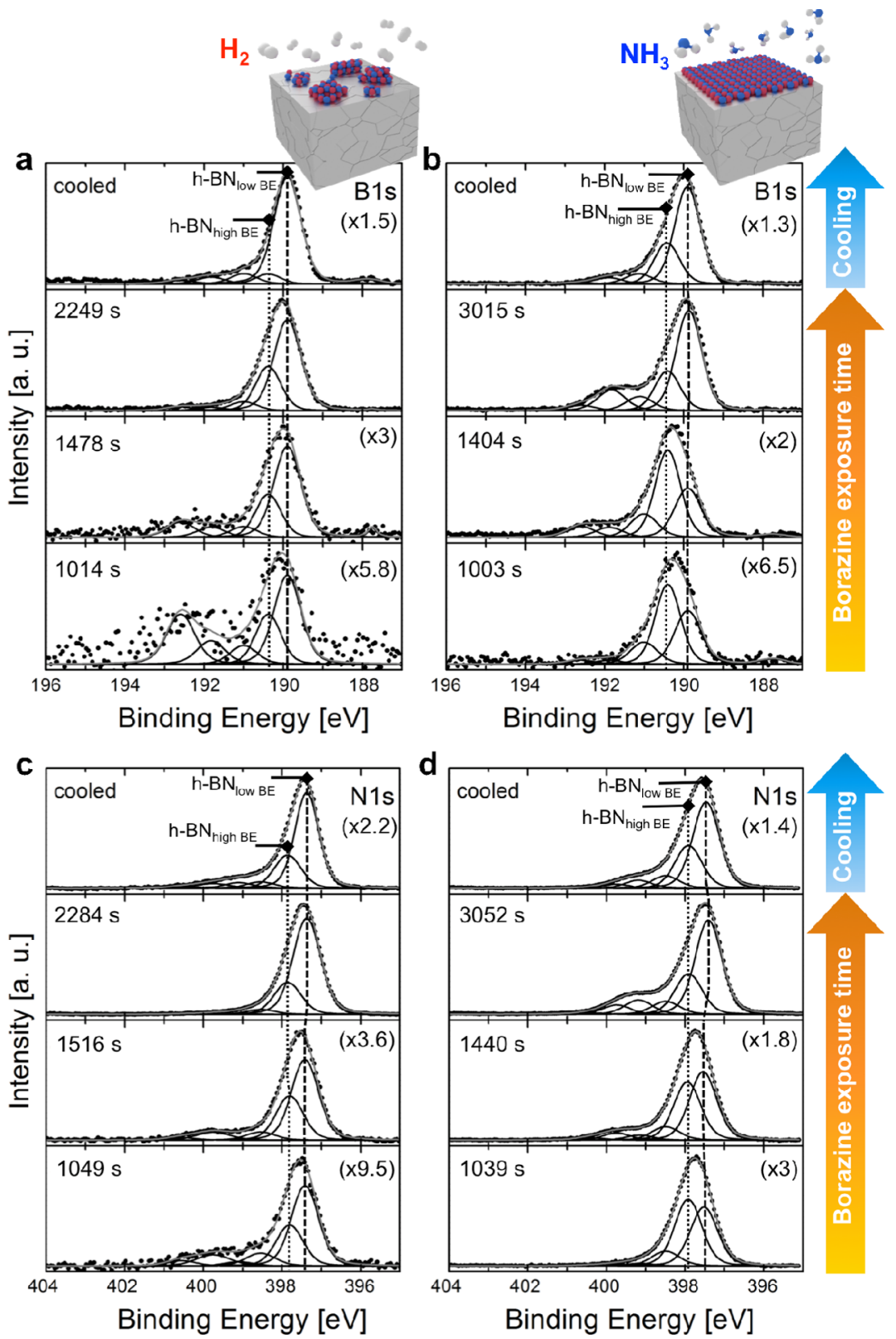

Figure 6. Time-resolved in situ XPS of the $\mathrm{B} 1 s$ core level for (a) $\mathrm{H}_{2}$ - and (b) $\mathrm{NH}_{3}$-preannealed Fe foil and the $\mathrm{N} 1 s$ core level for (c) $\mathrm{H}_{2}-$ and $(\mathrm{d})$ $\mathrm{NH}_{3}$-preannealed $\mathrm{Fe}$ foil during borazine exposure and initial cooling stage $\left(\sim 900{ }^{\circ} \mathrm{C}, \sim 1 \times 10^{-4} \mathrm{mbar}\right.$ for $\mathrm{NH}_{3}$-preannealed sample and $\sim 1 \times 10^{-3}$ mbar for $\mathrm{H}_{2}$-preannealed sample, $\left.\sim 10 \mathrm{~min}\right)$. Spectra are collected in normal emission geometry at photon energies of $620 \mathrm{eV}\left(\lambda_{\text {escape }} \approx 13 \AA\right)$.

pattern and film edge close-up (top left and bottom right insets, respectively), demonstrating the crystalline and monolayer properties of the material. An optical image of h-BN domains transferred onto a $\mathrm{SiO}_{2}(300 \mathrm{~nm}) / \mathrm{Si}$ wafer, acquired with a green filter to enhance the contrast with the substrate, is shown in Figure 4e. The Raman spectrum from the area indicated by the red dot (Figure 4f) exhibits a peak at $1369 \mathrm{~cm}^{-1}$, in agreement with literature values for CVD h-BN. ${ }^{43}$

In order to elucidate the growth mechanisms responsible for the differing h-BN morphologies observed in Figure 2, we employ in situ XRD and in situ XPS during growth. Figure 5 compares in situ XRD patterns acquired during $\mathrm{H}_{2}$ - (a) and $\mathrm{NH}_{3}$-preannealed (b) h-BN growth. The as-loaded catalyst foils display reflections corresponding to body-centered-cubic (bcc) $\alpha$-Fe. For the $\mathrm{H}_{2}$ preannealing (Figure 5a), the foils transform to face-centered-cubic (fcc) $\gamma$-Fe upon heating and con- currently grain growth occurs (shown via a sharpening of the reflections). We note that in our experimental setup the large Fe grain sizes $(\sim 80 \mu \mathrm{m}$ estimated from SEM) result in the diffraction pattern originating from only a few grains, and hence, the measured apparent texture directions are not necessarily representative of the texture of the entire foil. Upon introduction of borazine following the $\mathrm{H}_{2}$ preannealing, a strong reflection at $18^{\circ}$ is observed, which is ascribed to isothermal growth of few-layer h-BN, accompanied by multiple sharp reflections at higher angles that can be attributed to the formation of a small amount of Fe-borides $\left(\mathrm{Fe}_{2} \mathrm{~B}\right.$, and possibly $\mathrm{FeB}$ ). Furthermore, the majority catalyst phase changes from $\gamma$ Fe to $\alpha$-Fe (with possibly small amounts of $\gamma$-Fe remaining). After cooling to room temperature, the catalyst state consists of mostly $\alpha$-Fe with some minor Fe-borides and possibly some $\gamma$ Fe. The observed Fe-boride formation and the isothermal $\gamma$-Fe 
$\rightarrow \alpha$-Fe transition are both indicative of $\mathrm{B}$ uptake into the $\mathrm{Fe}$ and are consistent with our recent work on $\mathrm{H}_{2}$-preannealed h$\mathrm{BN}$ growth on Fe films. ${ }^{34}$

In contrast, the $\mathrm{NH}_{3}$ preannealing (Figure $5 \mathrm{~b}$ ) leads to a very different evolution: upon preannealing in $\mathrm{NH}_{3}$ the majority catalyst phase changes from $\alpha$-Fe to $\gamma$-Fe (as with $\mathrm{H}_{2}$ preannealing). However, although the XRD pattern for $\mathrm{NH}_{3}$ preannealing can be fully assigned to $\gamma$-Fe, we note that the comparably broad peak at $\sim 30^{\circ}$ could also correspond to the highest intensity reflection of $\varepsilon-\mathrm{Fe}_{3} \mathrm{~N}$ (not observed for $\mathrm{H}_{2}$ preannealing), which requires $\mathrm{N}$ uptake into the Fe foil. We corroborate such $\mathrm{N}$ uptake by control experiments on $\mathrm{Fe}(250$ $\mathrm{nm}) / \mathrm{SiO}_{2}(300 \mathrm{~nm}) / \mathrm{Si}$ which are exposed to the same $\mathrm{NH}_{3}$ preannealing annealing conditions. Although trace levels of diffused $\mathrm{Si}$ have to be considered for these films, ${ }^{34}$ the lower degree of texture allows Rietveld refinement of lattice constants. Upon $\mathrm{NH}_{3}$ exposure over a vacuum baseline, we find a lattice expansion corresponding to 0.6 atom $\% \mathrm{~N}$ uptake ${ }^{44}$ into the bulk of the $\gamma$-Fe films (Figure S5), confirming that $\mathrm{N}$ dissolves into the $\mathrm{Fe}$ under our $\mathrm{NH}_{3}$ preannealing conditions. When borazine is introduced, the preceding $\mathrm{NH}_{3}$ preannealing impacts on the further catalyst evolution: in contrast to the $\mathrm{H}_{2}$-preannealed foil, $\gamma$-Fe remains the majority catalyst phase and neither Fe-borides are formed nor is a $\gamma$-Fe $\rightarrow \alpha$-Fe transition observed during borazine exposure. Additionally, we do not observe the emergence of a few-layer h-BN related reflection at $\sim 18^{\circ}$. This lack of a signal corresponding to thick $\mathrm{h}-\mathrm{BN}$ films is consistent with the exclusive monolayer h-BN growth observed in our ex situ characterization above (we note that our XRD setup cannot detect monolayer h-BN; ${ }^{35}$ however, monolayer h-BN growth was confirmed by ex situ SEM for the $\mathrm{NH}_{3}$-preannealed sample grown during in situ XRD). During cooling to room temperature, after borazine exposure, a $\gamma$ - $\mathrm{Fe} \rightarrow$ $\alpha$-Fe transition ${ }^{44}$ occurs. The additional emergence of $\mathrm{Fe}_{4} \mathrm{~N}$ reflections upon cooling (which were not observed in the $\mathrm{H}_{2}$ preannealing data) further corroborates $\mathrm{N}$ uptake into the catalyst during the $\mathrm{NH}_{3}$-preannealed h-BN CVD. $\mathrm{Fe}_{4} \mathrm{~N}$ is only thermodynamically stable below $680{ }^{\circ} \mathrm{C},{ }^{44}$ which explains why $\mathrm{Fe}_{4} \mathrm{~N}$ only nucleates upon cooling.

In situ XPS data summarized in Figure 6 provides complementary, surface-sensitive information on the h-BN nucleation and growth processes. We note that the assignment of all the XPS peaks and their corresponding shifts is not trivial for such a complex multicomponent system. Hence, we focus here on the main signatures of h-BN CVD and the major differences arising from the two preannealings. Figure $6 \mathrm{a}-\mathrm{d}$ show the time-resolved evolution of the XP B1s and N1s core level spectra for a Fe foil preannealed at $\sim 900{ }^{\circ} \mathrm{C}$ in either $\mathrm{H}_{2}$ $(\mathrm{a}, \mathrm{c})$ or $\mathrm{NH}_{3}(\mathrm{~b}, \mathrm{~d})$ and subsequently exposed to borazine. The borazine exposure time (measured from the time at which the desired exposure pressure is reached) for each scan prior to cooling is indicated in the top left corner of each frame. Two main peak pairs are observed for both preannealings and are related to the h-BN structure, where the $\mathrm{B}$ atom is bonded to three $\mathrm{N}$ atoms in a planar hexagonal configuration. ${ }^{45}$ The $\mathrm{B} / \mathrm{N}$ peak pair with higher binding energies (BE) are centered at $190.5 / 397.9 \mathrm{eV}$ and the lower BE pair at $189.9 / 397.5 \mathrm{eV}$. Both pairs can relate to monolayer $\mathrm{h}-\mathrm{BN}$ and can arise due to differences in coupling between the h-BN layer and the catalyst. In particular, effects such as different grain orientations, intercalation, and rippling/restructuring of the surface are known to change the interaction of the overlying $2 \mathrm{D}$ film with the substrate. ${ }^{35}$ The increase in the intensity of the lower BE pair is then tentatively attributed to few-layer h-BN: ${ }^{46}$ on the basis of our previous work on $\mathrm{Cu}$-catalyzed h-BN CVD, ${ }^{35}$ we note that although the first h-BN layer in direct contact with the catalyst does show such coupling effects, for few-layer h-BN this interaction can be screened. Hence, an increasing peak intensity of the lower $\mathrm{BE}$ pair here can be indicative of the presence of few-layer h-BN. For the $\mathrm{H}_{2}$-preannealed foil during the initial exposure to borazine, the peaks of both the high and low BE pairs appear concurrently, with the low BE pair dominating. This is consistent with our SEM images showing the growth of multilayered islands for very short exposure times. With continuing borazine exposure, the relative peak intensity of the lower $\mathrm{BE}$ pair continues to rise, indicative of a thickening of the h-BN domains, and then increases even further upon cooling. An ex situ SEM image corresponding to this sample is shown in Figure S6a. In contrast, for the $\mathrm{NH}_{3}$ preannealed foil, the high $\mathrm{BE}$ pair is more intense in the early stages of growth, relative to the $\mathrm{H}_{2}$ preannealing, indicating isothermal growth of predominantly monolayer h-BN on Fe. The fact that the low BE pair dominates for the rest of the growth suggests that few-layer h-BN evolves with extended exposure time, which is indeed observed in the ex situ SEM image of this sample taken after growth and cooling (Figure S6b). Though the XPS growth results differ somewhat from those of our well-calibrated reactor and the optimized growths in Figure $2 \mathrm{~d}-\mathrm{f}$, we attribute this to the different conditions in the XPS chamber, notably the much lower base pressure $\left[\sim 10^{-9} \mathrm{mbar}\right]$ and the lower permissible pressure of $\mathrm{NH}_{3}[\sim 0.5$ mbar]. Effectively, the in situ XPS experiments resemble the CVD growth with an extended Stage 2, where insufficient Nenrichment of the catalyst bulk causes the formation of h-BN multilayers. We note that the slightly different ratio of the two $\mathrm{BE}$ components between the $\mathrm{B} 1 s$ and the corresponding $\mathrm{N} 1 s$ (bottom two frames, Figure 6) for the $\mathrm{NH}_{3}$ case can be attributed to the fact that the N1s spectra were always acquired after the B1s spectra, that is, with a typical time lag of $1 \mathrm{~min}$, the $\mathrm{N} 1 s$ spectra thus correspond to a later stage of the growth. The increase in the intensity of the two peak pairs with extended borazine exposure (as apparent from the different intensity scalings of each frame) confirms that, regardless of the preannealing, h-BN formation occurs isothermally, which is in agreement with our in situ XRD measurements and ex situ SEM observations. We further note that for the $\mathrm{H}_{2}$ preannealing case, a minor contribution from bulk precipitation upon cooling is also observed (Figure $3 \mathrm{a}-\mathrm{c}$ ). Conversely, for the $\mathrm{NH}_{3}$ preannealing case no signatures of precipitation are observed, even for very slow cooling rates (Figure $3 \mathrm{~d}-\mathrm{f}$ ).

Figure 7a,b show depth-resolved $\mathrm{B} 1 s$ core level spectra taken after borazine exposure and cooling for $\mathrm{H}_{2}-$ and $\mathrm{NH}_{3}$ preannealed foils, respectively. By changing the incident X-ray energies, and hence the inelastic mean free path of the photoelectrons $\left(\lambda_{\text {escape }}\right)$, the information depth can be varied, giving a more surface sensitive spectrum $\left(\lambda_{\text {escape }} \approx 10 \AA\right)$ for $h v$ $=400 \mathrm{eV}$ and a more bulk sensitive $\left(\lambda_{\text {escape }} \approx 13 \AA\right)$ spectrum for $h v=640 \mathrm{eV}$. For the $\mathrm{H}_{2}$-preannealed foil, we assign the component at $\sim 188 \mathrm{eV}$ to $\mathrm{B}$-related species (dissolved $\mathrm{B}$ or borides $)^{46-50}$ and note that this component does not have a corresponding pair in the N1s spectrum, as would be expected. Comparison of the relative intensities of this component shows that the B-related species are stronger in the more bulk sensitive spectra (Figure 7a inset), which is consistent with our $\mathrm{XRD}$ analysis, where we demonstrate that species present at higher concentrations in the catalyst bulk can segregate toward 


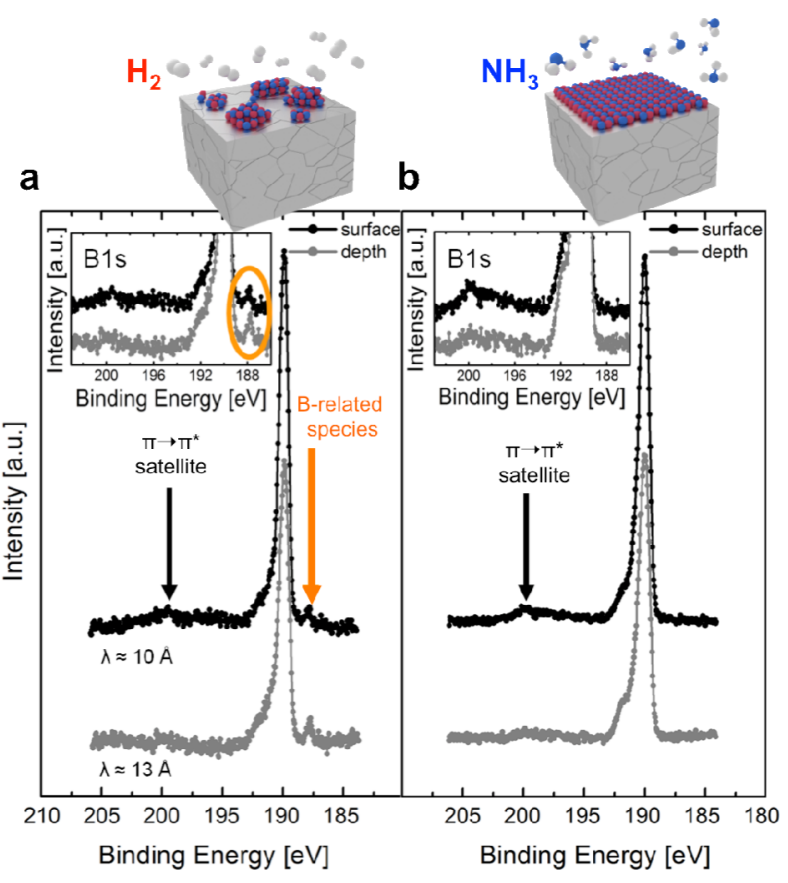

Figure 7. Depth-resolved in situ XPS B1s core level lines measured at room temperature in vacuum for (a) $\mathrm{H}_{2}$ - and (b) $\mathrm{NH}_{3}$-preannealed $\mathrm{Fe}$ foil after borazine exposure and cooling (growth parameters: $\sim 900{ }^{\circ} \mathrm{C}$, $\sim 1 \times 10^{-4} \mathrm{mbar}_{\text {for }} \mathrm{NH}_{3}$-preannealed sample and $\sim 1 \times 10^{-3} \mathrm{mbar}$ for $\mathrm{H}_{2}$-preannealed sample, $\sim 10 \mathrm{~min}$ borazine exposure time). The spectra are collected at photon energies of $400 \mathrm{eV}$ (surface sensitive; $\lambda_{\text {escape }} \approx 10 \AA$ ) and $640 \mathrm{eV}$ (bulk sensitive; $\lambda_{\text {escape }} \approx 13 \AA$ ).

the surface during cooling, leading to the formation of additional phases (i.e., borides for $\mathrm{H}_{2}$ preannealing and nitrides for $\mathrm{NH}_{3}$ preannealing). Interestingly, the component at $\sim 188$ $\mathrm{eV}$ is not detected for the $\mathrm{NH}_{3}$-preannealed foil, neither in the surface sensitive scan nor in the more bulk sensitive scan (Figure 7b). This is consistent with the B uptake being significantly reduced by the presence of $\mathrm{N}$ dissolved in the $\mathrm{Fe}$ bulk from the $\mathrm{NH}_{3}$ preannealing step. Both preannealings lead to the appearance of a small peak at $\sim 200 \mathrm{eV}$, which has been attributed to the $\pi-\pi^{*}$ plasmon shake up satellite corresponding to $\mathrm{sp}^{2}$ bonded hexagonal boron nitride. ${ }^{51-53}$ This satellite is not detected for c-BN. ${ }^{53}$ The sharper satellite peak for the $\mathrm{NH}_{3}$-preannealed sample attests to the higher quality of the h$\mathrm{BN}$ grown (Figure $7 \mathrm{~b}$ inset).

We also find that the onset of h-BN growth is strongly dependent on the borazine partial pressure, as summarized in Figure S7. For a given constant temperature, upon introducing $\sim 1 \times 10^{-4}$ mbar of borazine, the N1s and B1s scans for both types of preannealed foils exhibit flat lines, indicating the absence of h-BN growth. After 9 min at $\sim 1 \times 10^{-4}$ mbar of borazine, the $\mathrm{N} 1 s$ and $\mathrm{B} 1 s$ scans for the $\mathrm{H}_{2}$-preannealed sample remain flat. Small N1s and B1s peaks only start appearing for an increased borazine partial pressure of $\sim 7 \times 10^{-4} \mathrm{mbar}$. For the $\mathrm{H}_{2}$-preannealed sample, significant h- $\mathrm{BN}$ growth is achieved by further increasing the borazine pressure to $\sim 1 \times 10^{-3} \mathrm{mbar}$. We note that the sample was exposed to $\sim 7 \times 10^{-4}$ mbar of borazine for $15 \mathrm{~min}$ before increasing the pressure to $\sim 1 \times$ $10^{-3} \mathrm{mbar}$, and then exposed to this pressure for a further 20 min. In contrast, after $3 \mathrm{~min}$ of borazine exposure at $\sim 1 \times 10^{-4}$ mbar of borazine, a notable peak appears in both $\mathrm{N} 1 s$ and $\mathrm{B} 1 s$ scans for the $\mathrm{NH}_{3}$-preannealed sample, which corresponds to h$\mathrm{BN}$ nucleation. Upon continued exposure $(6 \mathrm{~min})$ at this same pressure, the peaks increase in intensity as the h-BN domains grow further. Combined, our in situ XRD and in situ XPS characterizations suggest a strong influence of $\mathrm{NH}_{3}$ preannealing not only on h-BN growth but also on the underlying catalyst phase evolution and $\mathrm{B} / \mathrm{N}$ uptake mechanisms.

Discussion. Our data reveals that the contribution of the $\mathrm{Fe}$ bulk reservoir is critical in determining the h-BN growth behavior. Although other catalysts offer much lower B and $\mathrm{N}$ solubilities such that precipitation-driven growth is minimized, our focus here on Fe substrates is motivated by our previous work, which shows $\mathrm{Fe}$ to be an excellent catalyst for highquality h-BN growth. ${ }^{34}$ In fact, we are able to use the bulk solubility of $\mathrm{B}$ and $\mathrm{N}$ in $\mathrm{Fe}$ as a key advantage, and hence

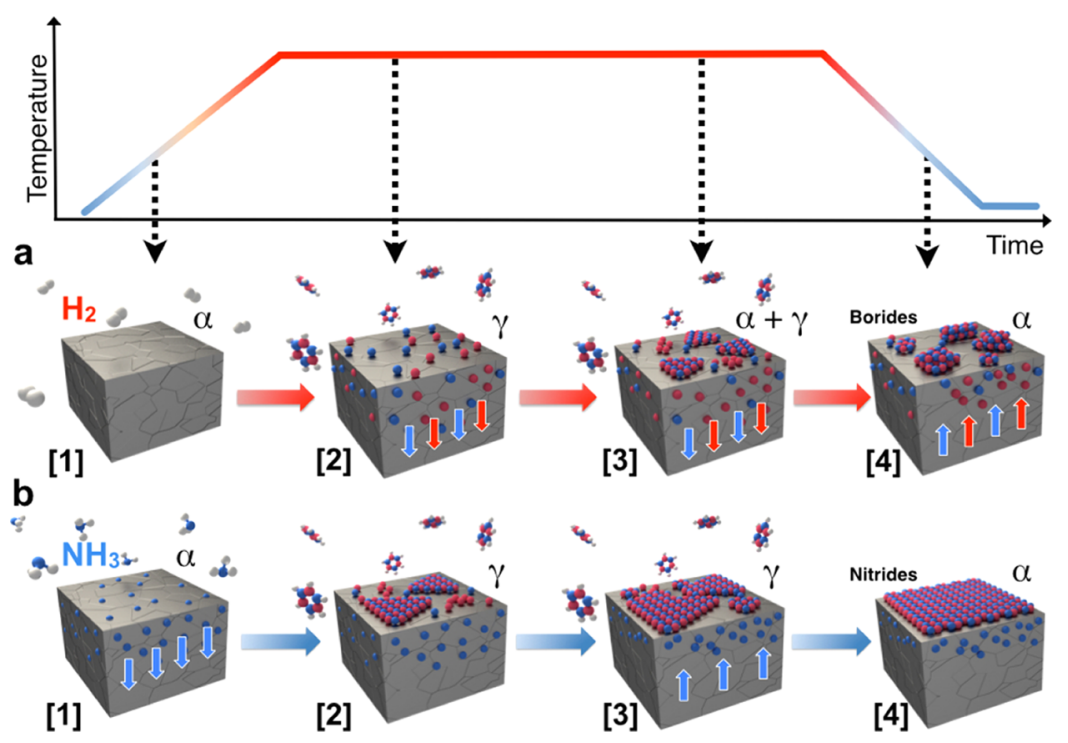

Figure 8. Schematic illustrating the near-surface region of the Fe catalyst and the proposed mechanisms involved in the growth of h-BN, comparing the (a) $\mathrm{H}_{2}$ - and (b) $\mathrm{NH}_{3}$-preannealed foils respectively during different CVD stages. The downward and upward arrows indicate species diffusing into and out of the catalyst, respectively. [1], [2], [3], and [4] represent the state of the catalyst during preannealing, short borazine exposure, long borazine exposure, and vacuum cooling, respectively. 
achieve better growth control using a bulk prefilling method, as discussed below. Similarly, a finite carbon solubility has previously been shown to substantially improve graphene growth uniformity on polycrystalline $\mathrm{Ni}$ and Co substrates, where the catalyst bulk acts as a mediating carbon sink that moderates variations in growth across different catalyst grains. $^{37,54}$

Figure 8 schematically summarizes the processes taking place during the salient stages of the CVD process on $\mathrm{Fe}$ foils, comparing the effects of the $\mathrm{H}_{2}$ and $\mathrm{NH}_{3}$ preannealing on the catalyst chemistry and on the growth of h-BN (Figure 8a,b), and interpreting them in the context of ternary phase diagram considerations (Figure 9). The two suggested growth pathways

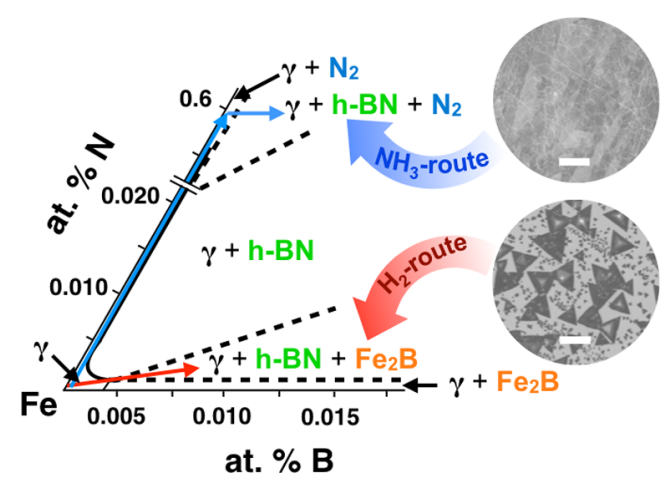

Figure 9. Fe-rich corner of the $\mathrm{Fe}-\mathrm{B}-\mathrm{N}$ phase diagram at $950{ }^{\circ} \mathrm{C}$ (adapted from Rogl and Schuster). ${ }^{55}$ The red and blue arrows represent the reaction pathway for the $\mathrm{H}_{2}$ - and $\mathrm{NH}_{3}$-preannealed foils respectively with the corresponding postgrowth SEM images of the sample surface. The scalebars are $20 \mu \mathrm{m}$.

are summarized in the Fe-rich corner of the $\mathrm{Fe}-\mathrm{B}-\mathrm{N}$ phase diagram at $950{ }^{\circ} \mathrm{C},{ }^{55}$ and are marked by the red and blue arrows, denoting the $\mathrm{H}_{2}$ and $\mathrm{NH}_{3}$ preannealing routes, respectively. The phase diagram is applicable to our experiments given that the changes in the phase boundaries between the sections at 950 and $1050{ }^{\circ} \mathrm{C}$ are minimal, ${ }^{55}$ and temperature uncertainties of $\pm 50{ }^{\circ} \mathrm{C}$ in commercial $\mathrm{CVD}$ reactors are not untypical. In the framework of our discussion, we assume that the $\mathrm{B}$ and $\mathrm{N}$ diffusivity does not vary significantly depending on their relative proportions but is only dependent on temperature.

CVD of a compound material, such as h-BN, requires simultaneous feeding of $\mathrm{B}$ and $\mathrm{N}$ species into the growing stoichiometric crystal, which presents a more complex scenario compared to graphene growth, where only $\mathrm{C}$ atoms need to be incorporated into the graphitic lattice. First, we outline the B and $\mathrm{N}$ fluxes necessary for h-BN CVD, involving precursor dissociation and the formation of h-BN domains. The impingement of borazine molecules and their dissociation provides a flux of $\mathrm{B}$ and $\mathrm{N}$ species at the catalyst surface. Concurrently, a flux of B and $\mathrm{N}$ dissolving into the catalyst bulk will deplete the surface. A net flux is required for h-BN growth, which is equal to the difference between the flux reaching the surface and that diffusing into the catalyst. During the initial stages of borazine dosing these two fluxes will be matched and no h-BN nucleation occurs (i.e., incubation period). However, the concentration of $\mathrm{B}$ and $\mathrm{N}$ at the surface will start to increase gradually until a critical supersaturation at the Fe surface is reached, giving rise to the first h-BN nucleation events. Following nucleation, growth of h-BN islands will proceed, fed by the net flux resulting from the precursor dissociation at the surface and the diffusion into the catalyst bulk. We briefly outline the importance of the bulk reservoir to CVD growth on catalysts with finite solubilities of the precursor species. ${ }^{37}$ For hBN CVD on catalysts with significant B and N solubilities (such as $\mathrm{Fe}$ ), the supply of $\mathrm{B}$ and $\mathrm{N}$ to the catalyst surface to feed h$\mathrm{BN}$ growth is mediated by their diffusion into the catalyst bulk. This allows uniform h-BN to be formed over the catalyst by locally saturating the catalyst close to the surface, while the bulk continues to provide a sink for B and $\mathrm{N}$ species. However, if the catalyst becomes saturated with these species throughout its bulk during the growth process, then the bulk of the catalyst no longer acts as a mediating sink and inhomogeneous few-layer h$\mathrm{BN}$ can readily form isothermally, as well as by the precipitation of $\mathrm{B}$ and $\mathrm{N}$ to the surface upon cool-down. How quickly the catalyst becomes saturated throughout its thickness is ultimately dictated by its permeability (i.e., the product of solubility and diffusivity). Thus, low permeabilities, relative to the rate of $\mathrm{B}$ and $\mathrm{N}$ delivery to the catalyst surface, favor a broad processing window for monolayer h-BN formation, and hence, it is highly desirable to be able to control this property. ${ }^{54}$

We first consider the $\mathrm{H}_{2}$ preannealing case. During preannealing up to $\sim 900{ }^{\circ} \mathrm{C}$ (Figure $8 \mathrm{a}[1]$ ), the $\mathrm{Fe}$ surface undergoes a reduction reaction of $\mathrm{Fe}$ oxides, ${ }^{56}$ formed from ambient air storage of the foils, accompanied by the phase transformation $\alpha$-Fe $\rightarrow \gamma$-Fe, as confirmed by the XRD data in Figure 5a. On precursor exposure, uptake of both $\mathrm{B}$ and $\mathrm{N}$ from the dissociation of borazine is confirmed by XRD, which shows the formation of borides and an expansion of the Fe lattice due to $\mathrm{N}$ dissolution for similar growth conditions. ${ }^{34}$ The catalyst reservoir is therefore partially filled by the constituent species, illustrated by the downward red and blue arrows in Figure $8 \mathrm{a}[2]$, and results in a longer incubation time for crystal growth because the critical supersaturation required for nucleation will take longer to achieve. This is consistent with the ternary $\mathrm{Fe}-$ $\mathrm{B}-\mathrm{N}$ phase diagram, in which the curve in the solvus for intermediate $\mathrm{B}: \mathrm{N}$ ratios is crossed at relatively high solubilies, and hence high permeabilities, of both species. At such intermediate $\mathrm{B}: \mathrm{N}$ ratios, it is not clear which of the species it is whose supply is the limiting factor that controls the growth. After $10 \mathrm{~min}$ of borazine exposure, the $\mathrm{XRD}$ data shows the appearance of the few-layer h-BN reflection at $\sim 18^{\circ}$, demonstrating that growth of additional h-BN layers occurs isothermally (small triangular domains in Figure 8a[3]). This is further confirmed by in situ XPS, which additionally reveals the concurrent appearance of h-BN mono- and multilayers (Figure $6 a, c)$.

Upon borazine removal and cooling (Figure 3b,c), we show that further nucleation of new domains and thickening of existing domains occurs for relatively slow cooling rates on $\mathrm{H}_{2}$ preannealed foils. The fact that the h-BN domain edges appear to be dissolving for a cooling rate of $50{ }^{\circ} \mathrm{C} / \mathrm{min}$ (Figure $3 \mathrm{c}$ inset) could be explained by considering that while the catalyst surface is saturated with $\mathrm{B}$ and $\mathrm{N}$ during growth, the catalyst is not saturated throughout the bulk (i.e., the $\mathrm{B}$ and $\mathrm{N}$ concentration is not uniform across the depth). Thus, the slow cooling rate is effectively equivalent to a postanneal, which can result in the increased diffusion of $\mathrm{B}$ and $\mathrm{N}$ into the catalyst bulk, decreasing the concentration of $\mathrm{B}$ and $\mathrm{N}$ near the catalyst surface and leading to the partial dissolution of the existing domains. For rapid quenching, a smaller contribution to growth from bulk precipitation is also expected (Figure $8 \mathrm{a}[4]$ ). We note that although we show clear evidence of $\mathrm{B}$ and $\mathrm{N}$ uptake 
in the Fe bulk, this does not necessarily imply precipitation into h-BN upon cooling because the assembly of an h-BN domain requires a stoichiometric arrangement of atoms in the hexagonal lattice. For monoelemental systems such as C, growth of a graphic lattice by precipitation on cooling is simpler because only one element needs to be incorporated at the growth front. For compound materials like h-BN, this becomes less trivial. However, our ex situ SEM indicates that precipitation-driven growth does in fact occur. This can be explained by noting that the high permeabilities of $\mathrm{B}$ and $\mathrm{N}$ that saturate the catalyst surface (evidenced by boride formation and $\mathrm{N}$-induced $\mathrm{Fe}$ lattice expansion in XRD, as well as dissolved $\mathrm{B} /$ borides in the Fe subsurface observed by in situ XPS) can lead to inhomogeneous isothermal multilayer growth, as well as further multilayer formation upon cooling. The compositional trajectory for the $\mathrm{H}_{2}$-preannealed foil can thus be summarized by the red arrow in Figure 9, which-starting in the $\gamma$-Fe phase field-crosses the boundary $\gamma$-Fe $\rightarrow \gamma$-Fe + h-BN $+\mathrm{Fe}_{2} \mathrm{~B}$ during simultaneous feeding of $\mathrm{N}$ and $\mathrm{B}$ from borazine dissociation. In terms of growth modes, the formation of h$\mathrm{BN}$ is predominantly isothermal, with a small contribution from bulk precipitation on immediate cooling, which is relatively minor given that the diffusivity of species rapidly decreases with temperature.

The chemical and structural changes in the Fe catalyst for the $\mathrm{NH}_{3}$-preannealed foil are markedly different. For the preannealed sample, we measure a lattice expansion of the $\mathrm{Fe}$ due to $\mathrm{N}$ uptake $^{44}$ corresponding to $\sim 0.6$ atom $\% \mathrm{~N}$ (downward blue arrows in Figure $8 \mathrm{~b}[1]$ ), and possibly due to the additional formation of $\varepsilon$ - $\mathrm{Fe}_{3} \mathrm{~N}$ as a minority phase. We note that the $\mathrm{Fe}-\mathrm{N}$ phase diagram does not predict the presence of $\varepsilon-\mathrm{Fe}_{3} \mathrm{~N}$ at the temperatures and $\mathrm{N}$ content of our experiment; therefore, the contribution to the small reflection at $\sim 30^{\circ}$ in Figure $5 \mathrm{~b}$ during preannealing is most likely due to $\gamma$-Fe. The presence of $\mathrm{N}$ in $\mathrm{Fe}$ has been shown to significantly reduce the $\alpha-\mathrm{Fe} \rightarrow \gamma$-Fe transformation temperature, ${ }^{44}$ from $912{ }^{\circ} \mathrm{C}$ for phase-pure $\mathrm{Fe}$ to $875{ }^{\circ} \mathrm{C}$ for $\mathrm{Fe}-0.6$ atom $\% \mathrm{~N}$, which is therefore in line with our XRD phase assignment. As a consequence of the $\mathrm{N}$ enrichment before borazine dosing, the $\mathrm{B}: \mathrm{N}$ ratio in the bulk of the foil is close to zero during dosing. Due to the shape of the solvus, crossing it to form h-BN only requires a small fraction of added B $(\sim 0.002$ at $\%$ B $){ }^{55}$ The fact that borides are not detected effectively means that the solubility, and thus permeability, of B is greatly reduced when the catalyst is saturated throughout with $\mathrm{N}$. With lower B permeability, the supersaturation required to form an h-BN nucleus therefore will be reached for lower borazine partial pressures compared to the $\mathrm{H}_{2}$-preannealed foil or for shorter exposure times at the same borazine exposure pressure. The first case is clearly supported by the in situ XPS measurements. As shown in Figure S7, the borazine partial pressure required for h-BN growth at $\sim 900{ }^{\circ} \mathrm{C}$ depends on the preannealing performed and is found to be $\sim 1 \times 10^{-3}$ mbar for the $\mathrm{H}_{2}$ preannealed foil, upon which growth is immediately observed, and $\sim 1 \times 10^{-4}$ mbar for the $\mathrm{NH}_{3}$-preannealed foil where h-BN begins to form after $3 \mathrm{~min}$ of borazine exposure. We also confirm a shorter incubation time under the same borazine exposure pressure for $\mathrm{NH}_{3}$-preannealed foils compared to $\mathrm{H}_{2}$ preannealed foils through SEM characterization (Figure 2a,d insets). The ex situ SEM images show that as we start dosing borazine, the nucleation of h-BN domains occurs much more rapidly on the $\mathrm{NH}_{3}$-preannealed foil, with triangular-shaped islands already observed after $14 \mathrm{~s}$ exposure (Figure $8 \mathrm{~b}[2]$ ). For longer exposure times the domains continue to grow laterally and neighboring domains start to coalesce. Contrary to the $\mathrm{H}_{2}$ preannealing case, we do not detect the isothermal $\gamma$-Fe phase transformation to $\alpha$-Fe. This, together with the lack of borides, both of which are processes linked to B uptake, ${ }^{34}$ reinforces the conclusion that the $\mathrm{NH}_{3}$-preannealing and the corresponding $\mathrm{N}$ uptake into the catalyst limits $\mathrm{B}$ diffusion into the $\mathrm{Fe}$ during subsequent borazine exposure, that is, given that the $\mathrm{B}$ and $\mathrm{N}$ solubilities are interdependent, the high $\mathrm{N}$ concentration in $\gamma$ $\mathrm{Fe}$ makes the corresponding $\mathrm{B}$ permeability very low. Additionally, the limited amount of B in the bulk reduces the likelihood of multilayer formation on cooling, as only $\mathrm{N}$ can escape from the sample surface. We note that during dosing at temperature, and in the absence of $\mathrm{NH}_{3}$ to replenish the bulk with $\mathrm{N}$, these species can diffuse back out to the surface where they can leave as $\mathrm{N}_{2}$ (upward blue arrows in Figure $8 \mathrm{~b}[3]$ ). Indeed, Figure $\mathrm{S} 3$ provides evidence demonstrating that the beneficial effect of the bulk $\mathrm{N}$ can be lost by long annealing times in vacuum prior to borazine exposure. Hence, to achieve uniform monolayer films, Stage 2 (Figure 1) should be kept as short as possible.

The XRD data in Figure $5 \mathrm{~b}$ illustrates that during growth, and subsequently during simultaneous cooling and borazine removal, we do not detect the appearance of the reflection at $\sim 18^{\circ}$ for the $\mathrm{NH}_{3}$ preannealing case, confirming that the h-BN thickness does not increase significantly. This is corroborated by ex situ SEM (Figure $2 \mathrm{~d}-\mathrm{f}$ ), which shows that the h-BN remains as monolayers for these growth conditions. Indeed, the SEM images in Figure $3 \mathrm{~d}-\mathrm{f}$ are further proof that multilayers do not appear during cooling, even for slow cooling rates, which typically allow sufficient time for species to segregate at the surface, indicating a lack of $B$ atoms that can be supplied from the bulk. The main difference compared with the $\mathrm{H}_{2}$ preannealing case is that $\mathrm{B}$ is now the limiting factor that governs the growth, which thus becomes kinetically controlled. ${ }^{37}$ In this regime, it therefore is possible to exclusively grow monolayer h-BN on a N-prefilled catalyst, as long as the extent of B diffusion into the catalyst bulk remains limited (Figure $8 \mathrm{~b}[4]$ ). On the basis of our experimental evidence, we propose that for the $\mathrm{NH}_{3}$-preannealed foil, the compositional pathway runs along the $\mathrm{Fe}-\mathrm{N}$ edge in the $\gamma$-Fe phase field during annealing (Figure 9, long blue arrow), because no B is supplied at this stage and only $\mathrm{N}$ dissolves into the Fe. Upon subsequent borazine dosing, the trajectory crosses the boundary into the $\gamma-\mathrm{Fe}+\mathrm{h}-\mathrm{BN}+\mathrm{N}_{2}$ phase field (short blue arrow) based on the isothermal growth of h-BN monolayers observed in combined SEM, XRD, and XPS.

Our current work shows that the general bulk-mediated growth model reported here is also applicable to graphene CVD on Fe foils using a $\mathrm{C}_{2} \mathrm{H}_{2}$ carbon source. Analogously to the h-BN growth, we observe reduced incubation times for $\mathrm{NH}_{3}$-preannealed foils (i.e., $\mathrm{N}$-filled bulk reservoir) compared to vacuum-annealed foils (unfilled bulk reservoir). The data will be presented in future work; however, it demonstrates the robustness and wide applicability of our model, which is anticipated to be relevant for the fabrication of heterostructures. Indeed, the use of a catalyst prefilling method for growth control has previously been used in other material systems. A notable example is the catalytic growth of $\mathrm{Si} / \mathrm{Ge}$ heterostructure nanowires (NWs) with compositionally abrupt interfaces, which requires the minimization of the solubility of $\mathrm{Si}$ and $\mathrm{Ge}$ in the liquid $\mathrm{Au}$ catalyst in order to reduce the catalyst bulk reservoir effect. $^{57,58}$ 
Conclusion. In summary, a significant level of improvement in the growth of h-BN is achieved through a bulk reservoir filling effect in an as-received $\mathrm{Fe}$ foil by predosing $\mathrm{N}$, one of the constituent species, in the form of $\mathrm{NH}_{3}$ during preannealing. Using in situ XRD and XPS, we demonstrate how $\mathrm{N}$-induced changes to the $\mathrm{Fe}$ catalyst phase evolution and composition directly impact the h-BN incubation time and the uptake of $\mathrm{B}$ and $\mathrm{N}$ species during dosing. These critical parameters then determine structural h-BN features, such as number of layers, domain size, and nucleation density.

When the catalyst bulk is enriched with $\mathrm{N}$ from the high temperature and high pressure preannealing in $\mathrm{NH}_{3}$, the diffusion of $\mathrm{B}$ and $\mathrm{N}$ species in the $\mathrm{Fe}$ subsurface during subsequent borazine exposure is limited. This effectively prevents significant additional h-BN layer formation that typically occurs by precipitation upon cooling, and which is indeed observed for $\mathrm{H}_{2}$-preannealed $\mathrm{Fe}$ foils (i.e., unfilled bulk reservoir). Bulk filling also leads to shorter incubation times and lower borazine partial pressures required to nucleate h-BN, which reduces the probability of multilayer formation and a large domain size distribution.

Preannealing the catalyst with $\mathrm{NH}_{3}$ allows us to control the subsequent uptake of precursor species during dosing. Importantly, given the interdependency of the $\mathrm{B}$ and $\mathrm{N}$ solubilities, it allows us to lower the permeability of B, which leads to uniform h-BN monolayer growth. The catalyst bulk prefilling method presented here therefore provides an elegant alternative to using different catalysts or using catalyst alloying to control the permeability of the growth species. The general model that we derive, based on complementary ex situ and in situ data, in combination with phase diagram considerations, forms a coherent picture of the key bulk contributions to growth control and, importantly, is applicable to other catalytically grown $2 \mathrm{D}$ materials.

Methods. h-BN Growth. As-received Fe foil $(0.1 \mathrm{~mm}$, Alfa Aesar, $99.99 \%$ purity) is used for all experiments. The h-BN domains and films are grown in a customized Aixtron BM3 cold-wall reactor (base pressure $1 \times 10^{-6} \mathrm{mbar}$ ). CVD growth of $\mathrm{h}-\mathrm{BN}$ is performed using a borazine $(\mathrm{HBNH})_{3}$ precursor at a temperature of $\sim 900^{\circ} \mathrm{C}$ and a total pressure of $6 \times 10^{-4} \mathrm{mbar}$. The samples are typically heated in 4 mbar of $\mathrm{NH}_{3}$ or $\mathrm{H}_{2}$ at 100 ${ }^{\circ} \mathrm{C} / \mathrm{min}$ up to $750{ }^{\circ} \mathrm{C}$ and then at $50{ }^{\circ} \mathrm{C} / \mathrm{min}$ up to $\sim 900{ }^{\circ} \mathrm{C}$. The estimated uncertainty in the temperature measurement is $\pm 25{ }^{\circ} \mathrm{C}$. Immediately after reaching $\sim 900{ }^{\circ} \mathrm{C}$ the $\mathrm{NH}_{3}$ or $\mathrm{H}_{2}$ is removed. Borazine is dosed into the chamber through a leak valve (from a liquid reservoir) and after growth (growth times varied from $14 \mathrm{~s}$ to $8 \mathrm{~min}$ ) the borazine leak valve is closed and the heater is turned off. Samples are cooled in vacuum.

Transfer. For Raman spectroscopy, optical microscopy, and DF-TEM/STEM, we transfer the h-BN via the electrochemical bubbling method. ${ }^{15}$ We perform the transfer by spin coating a support layer of poly(methyl methacrylate) (PMMA) at 5000 $\mathrm{rpm}$ for $40 \mathrm{~s}$ onto the h-BN. The sample is placed in a $\mathrm{NaOH}$ bath $(1 \mathrm{M})$ and during electrolysis $\mathrm{H}_{2}$ bubbles evolve at the $\mathrm{h}$ $\mathrm{BN} / \mathrm{Fe}$ interface, lifting the film from the substrate. The $\mathrm{PMMA} / \mathrm{h}-\mathrm{BN}$ film is rinsed in deionized (DI) water and scooped onto a $\mathrm{SiO}_{2}(300 \mathrm{~nm}) / \mathrm{Si}$ wafer where it is left to dry. The PMMA is removed by immersing the sample in acetone for $\sim 12 \mathrm{~h}$, followed by a rinse in IPA.

Characterization. For the ex situ characterization of the h$\mathrm{BN}$ on the catalyst, we use scanning electron microscopy (SEM, Zeiss SigmaVP, $2 \mathrm{kV}$ ). Optical images are acquired using a Nikon eclipse ME600L microscope and a green filter was introduced for enhanced contrast. Raman spectroscopy is performed with a Renishaw Raman InVia microscope using a $50 \times$ objective lens and a $532 \mathrm{~nm}$ laser excitation. A Philips CM200 was used for bright-field (BF-) and dark-field transmission electron microscopy (DF-TEM) and selected area electron diffraction (SAED) at $80 \mathrm{kV}$. A Nion UltraSTEM 100 was employed for scanning transmission electron microscopy (STEM), using an electron acceleration voltage of $60 \mathrm{kV}$ and a high angle annular dark field (HAADF) detector. Atomic-resolution STEM data was processed to reduce contributions from probe-tails (an unprocessed STEM image is shown in Figure S4). ${ }^{39}$ The intensity profile was extracted from the processed image data by subtracting the remaining averaged intensity at intensity minima between atoms from the profile followed by normalizing the intensity at $\mathrm{B}$ sites to 1. As grown h-BN films were transferred from the catalyst for S(TEM) via the bubbling method onto holey carbon TEM grids with regular hole arrays.

In situ X-ray diffraction (XRD) was measured at the European Synchrotron Research Facility (beamline BM20/ ROBL) using a X-ray wavelength of $1.078 \AA$ in a previously described setup. ${ }^{30,34}$ Measurements were acquired in symmetric Theta-2Theta geometry (information depth in $\mu \mathrm{m}$ range) with the $\mathrm{Fe}$ foils clamped on one side by alumina spacers. The intensity step at $\sim 16^{\circ}$ in all measurements is due to the arrangement of detector and X-ray entrance/exit slits into the reaction chamber. We note that reflection positions shift between room temperature and CVD temperature scans due to thermal expansion. For phase identification the Inorganic Crystal Structure Database (ICSD) $(\alpha$-Fe, 53451; $\gamma$-Fe, 44862; $\mathrm{Fe}_{2} \mathrm{~B}, 391330 ; \mathrm{FeB}, 391331 ; \varepsilon-\mathrm{Fe}_{3} \mathrm{~N}, 80930 ; \gamma^{\prime}-\mathrm{Fe}_{4} \mathrm{~N}, 79980 ; \mathrm{h}-$ $\mathrm{BN}, 167799)$ and the International Center for Diffraction Data (ICDD) database $\left(\mathrm{Fe}_{2} \mathrm{~B}, 361332 ; \mathrm{FeB}, 320463\right)$ were used. Rietveld refinement of data was done using X'Pert Plus software. Quoted in situ XRD temperatures $\left(\sim 900^{\circ} \mathrm{C}\right)$ may be underestimated by up to $25^{\circ} \mathrm{C}$, thus explaining the observed $\alpha$ $\mathrm{Fe} \rightarrow \gamma$-Fe transition for the $\mathrm{H}_{2}$ pretreatment (Figure 5a), which would be thermodynamically only expected for $>912{ }^{\circ} \mathrm{C}$ for pure $\mathrm{Fe}^{44}$

In situ high-pressure XPS measurements during preannealing, growth, and cooling were performed at the ISISS end station of the FHI-MPG at the BESSY II synchrotron. We employ a high-pressure setup that consists of a cell (base pressure $\approx 10^{-9}$ mbar) that is attached to a set of differentially pumped electrostatic lenses and a differentially pumped analyzer (Phoibos 150, SPECS GmbH). All the spectra are acquired in normal emission geometry, using a spot size of 80 $\mu \mathrm{m} \times 150 \mu \mathrm{m}$ and with a spectral resolution of $\sim 0.3 \mathrm{eV}$. To perform depth-resolution experiments, the photon energy $\left(E_{\text {photon }}\right)$ is varied in order to change the kinetic energy of the emitted photoelectrons, thus changing the inelastic mean free paths, $\lambda_{\text {escape }}$. All spectra are background-corrected (Shirley) and analyzed using Doniach-Sùnjić functions convoluted with Gaussian profiles with an accuracy of $\sim 0.05$ $\mathrm{eV}$. All binding energies are referenced to measured Fermi edges.

\section{ASSOCIATED CONTENT}

\section{S Supporting Information}

The Supporting Information is available free of charge on the ACS Publications website at DOI: 10.1021/acs.nanolett.5b04586. 
Further characterization of h-BN, illustrating the preannealing-independent distribution of domains across the polycrystalline foils; bimodal domains size distribution for $\mathrm{H}_{2}$-preannealing; effect of extended Stage 2 on h$\mathrm{BN}$ morphology; STEM image of monolayer h-BN sputtering; Fe lattice expansion as a function of temperature and annealing atmosphere; ex situ SEM images of the in situ XPS samples in Figure 6 and pressure-dependent growth measured by in situ XPS. (PDF)

\section{AUTHOR INFORMATION}

\section{Corresponding Author}

*E-mail:sh315@cam.ac.uk.

\section{Notes}

The authors declare no competing financial interest.

\section{ACKNOWLEDGMENTS}

S.C. and R.W. acknowledge funding from EPSRC (Doctoral training award). R.S.W. acknowledges a Research Fellowship from St. John's College, Cambridge and a EU Marie Skłodowska-Curie Individual Fellowship (Global) under grant ARTIST (no. 656870) from the European Union's Horizon 2020 research and innovation programme. B.C.B. acknowledges funding from the European Union's Horizon 2020 research and innovation programme under the Marie Skłodowska-Curie grant agreement No 656214-2DInterFOX. B.C.B. and J.C.M. acknowledge support from the Austrian Science Fund (FWF): P25721-N20 and the Austrian Research Promotion Agency (FFG): 848152-GraphenMoFET. A.C.-V. acknowledges the Conacyt Cambridge Scholarship and Roberto Rocca Fellowship. S.H. acknowledges funding from ERC grant InsituNANO (no. 279342). We acknowledge the European Synchrotron Radiation Facility (ESRF) for provision of synchrotron radiation facilities at the BM20/ROBL beamline. We acknowledge the Helmholtz-Zentrum-Berlin Electron storage ring BESSY II for provision of synchrotron radiation at the ISISS beamline. We thank the ESRF and BESSY staff for continued support of our experiments and valuable discussion.

\section{REFERENCES}

(1) Lin, Y.; Connell, J. W. Nanoscale 2012, 4, 6908-6939.

(2) Pakdel, A.; Bando, Y.; Golberg, D. Chem. Soc. Rev. 2014, 43, 934-959.

(3) Song, X.; Hu, J.; Zeng, H. J. Mater. Chem. C 2013, 1, 2952-2969.

(4) Novoselov, K. S.; Fal'ko, V. I.; Colombo, L.; Gellert, P. R.; Schwab, M. G.; Kim, K. Nature 2012, 490, 192-200.

(5) Fiori, G.; Bonaccorso, F.; Iannaccone, G.; Palacios, T.; Neumaier, D.; Seabaugh, A.; Banerjee, S. K.; Colombo, L. Nat. Nanotechnol. 2014, 9, 768-779.

(6) Butler, S. Z.; Hollen, S. M.; Cao, L.; Cui, Y.; Gupta, J. A.; Gutierrez, H. R.; Heinz, T. F.; Hong, S. S.; Huang, J.; Ismach, A. F.; Johnston-halperin, E.; Kuno, M.; Plashnitsa, V. V.; Robinson, R. D.; Ruoff, R. S.; Salahuddin, S.; Shan, J.; Shi, L.; Spencer, O. M. G.; Terrones, M.; Windl, W.; Goldberger, J. E. ACS Nano 2013, 7, 28982926.

(7) Hofmann, S.; Braeuninger-Weimer, P.; Weatherup, R. S. J. Phys. Chem. Lett. 2015, 6, 2714-2721.

(8) Nørskov, J. K.; Bligaard, T.; Rossmeisl, J.; Christensen, C. H. Nat. Chem. 2009, 1, 37-46.

(9) Mattevi, C.; Kim, H.; Chhowalla, M. J. Mater. Chem. 2011, 21, 3324-3334.

(10) Park, S.; Lee, J.; Kim, H. S.; Park, J.-B.; Lee, K. H.; Han, S. A.; Hwang, S.; Kim, S.-W.; Shin, H.-J. ACS Nano 2015, 9, 633-638.
(11) Kim, K. K.; Hsu, A.; Jia, X.; Kim, S. M.; Shi, Y.; Hofmann, M.; Nezich, D.; Rodriguez-Nieva, J. F.; Dresselhaus, M.; Palacios, T.; Kong, J. Nano Lett. 2012, 12, 161-166.

(12) Song, L.; Ci, L.; Lu, H.; Sorokin, P. B.; Jin, C.; Ni, J.; Kvashnin, A. G.; Kvashnin, D. G.; Lou, J.; Yakobson, B. I.; Ajayan, P. M. Nano Lett. 2010, 10, 3209-3215.

(13) Park, J.-H.; Park, J. C.; Yun, S. J.; Kim, H.; Luong, D. H.; Kim, S. M.; Choi, S. H.; Yang, W.; Kong, J.; Kim, K. K.; Lee, Y. H. ACS Nano 2014, 8, 8520-8528.

(14) Kim, G.; Jang, A.-R.; Jeong, H. Y.; Lee, Z.; Kang, D. J.; Shin, H. S. Nano Lett. 2013, 13, 1834-1839.

(15) Gao, L.; Ren, W.; Xu, H.; Jin, L.; Wang, Z.; Ma, T.; Ma, L.-P.; Zhang, Z.; Fu, Q.; Peng, L.-M.; Bao, X.; Cheng, H.-M. Nat. Commun. 2012, 3, 699.

(16) Oshima, C.; Itoh, a.; Rokuta, E.; Tanaka, T.; Yamashita, K.; Sakurai, T. Solid State Commun. 2000, 116, 37-40.

(17) Shi, Y.; Hamsen, C.; Jia, X.; Kim, K. K.; Reina, A.; Hofmann, M.; Hsu, A. L.; Zhang, K.; Li, H.; Juang, Z.-Y.; Dresselhaus, M. S.; Li, L.-J.; Kong, J. Nano Lett. 2010, 10, 4134-4139.

(18) Orofeo, C. M.; Suzuki, S.; Hibino, H. J. Phys. Chem. C 2014, $118,3340-3346$.

(19) Vinogradov, N. A.; Zakharov, A. A.; Ng, M. L.; Mikkelsen, A.; Lundgren, E.; Mårtensson, N.; Preobrajenski, A. B. Langmuir 2012, 28, $1775-1781$.

(20) Morscher, M.; Corso, M.; Greber, T.; Osterwalder, J. Surf. Sci. 2006, 600, 3280-3284.

(21) Sutter, P.; Lahiri, J.; Albrecht, P.; Sutter, E. ACS Nano 2011, 5, 7303-7309.

(22) Simonov, K. A.; Vinogradov, N. A.; Ng, M. L.; Vinogradov, A. S.; Mårtensson, N.; Preobrajenski, A. B. Surf. Sci. 2012, 606, 564-570.

(23) Corso, M.; Auwärter, W.; Muntwiler, M.; Tamai, A.; Greber, T.; Osterwalder, J. Science 2004, 303, 217-220.

(24) Chatterjee, S.; Luo, Z.; Acerce, M.; Yates, D. M.; Johnson, A. T. C.; Sneddon, L. G. Chem. Mater. 2011, 23, 4414-4416.

(25) Auwärter, W.; Kreutz, T. J.; Greber, T.; Osterwalder, J. Surf. Sci. 1999, 429, 229-236.

(26) Li, Q.; Liu, M.; Zhang, Y.; Liu, Z. Small 2016, 12, 32-50.

(27) Sutter, P.; Huang, Y.; Sutter, E. Nano Lett. 2014, 14, 48464851.

(28) Liu, Z.; Ma, L.; Shi, G.; Zhou, W.; Gong, Y.; Lei, S.; Yang, X.; Zhang, J.; Yu, J.; Hackenberg, K. P.; Babakhani, A.; Idrobo, J.-C.; Vajtai, R; Lou, J.; Ajayan, P. M. Nat. Nanotechnol. 2013, 8, 119-124.

(29) Tay, R. Y.; Griep, M. H.; Mallick, G.; Tsang, S. H.; Singh, R. S.; Tumlin, T.; Teo, E. H. T.; Karna, S. P. Nano Lett. 2014, 14, 839-846.

(30) Bayer, B. C.; Baehtz, C.; Kidambi, P. R.; Weatherup, R. S.; Mangler, C.; Kotakoski, J.; Goddard, C. J. L.; Caneva, S.; CabreroVilatela, A.; Meyer, J. C.; Hofmann, S. Appl. Phys. Lett. 2014, 105, 143111.

(31) Fouquet, M.; Bayer, B. C.; Esconjauregui, S.; Thomsen, C.; Hofmann, S.; Robertson, J. J. Phys. Chem. C 2014, 118, 5773-5781.

(32) Ahmad, P.; Khandaker, M. U.; Amin, Y. M.; Muhammad, N.; Usman, A. R.; Amin, M. New J. Chem. 2015, 39, 7912-7915.

(33) Hu, B.; Jin, Y.; Guan, D.; Luo, Z.; Shang, B.; Fang, Q.; Ruan, H. J. Phys. Chem. C 2015, 119, 24124-24131.

(34) Caneva, S.; Weatherup, R. S.; Bayer, B.; Brennan, B.; Spencer, S. J.; Mingard, K.; Cabrero-Vilatela, A.; Baehtz, C.; Pollard, A. J.; Hofmann, S. Nano Lett. 2015, 15, 1867-1875.

(35) Kidambi, P. R.; Blume, R.; Kling, J.; Wagner, J. B.; Baehtz, C.; Weatherup, R. S.; Schloegl, R.; Bayer, B. C.; Hofmann, S. Chem. Mater. 2014, 26, 6380-6392.

(36) Li, X.; Cai, W.; Colombo, L.; Ruoff, R. S. Nano Lett. 2009, 9, 4268-4272.

(37) Weatherup, R. S.; Dlubak, B.; Hofmann, S. ACS Nano 2012, 6, 9996-10003.

(38) Artyukhov, V.; Hao, Y.; Ruoff, R.; Yakobson, B. Phys. Rev. Lett. 2015, 114, 115502.

(39) Krivanek, O. L.; Chisholm, M. F.; Nicolosi, V.; Pennycook, T. J.; Corbin, G. J.; Dellby, N.; Murfitt, M. F.; Own, C. S.; Szilagyi, Z. S.; 
Oxley, M. P.; Pantelides, S. T.; Pennycook, S. J. Nature 2010, 464, 571-574.

(40) Jin, C.; Lin, F.; Suenaga, K.; Iijima, S. Phys. Rev. Lett. 2009, 102, $3-6$.

(41) Meyer, J. C.; Chuvilin, A.; Algara-Siller, G.; Biskupek, J.; Kaiser, U. Nano Lett. 2009, 9, 2683-2689.

(42) Warner, J. H.; Rümmeli, M. H.; Bachmatiuk, A.; Büchner, B. ACS Nano 2010, 4, 1299-1304.

(43) Gorbachev, R. V.; Riaz, I.; Nair, R. R.; Jalil, R.; Britnell, L.; Belle, B. D.; Hill, E. W.; Novoselov, K. S.; Watanabe, K.; Taniguchi, T.; Geim, A. K.; Blake, P. Small 2011, 7, 465-468.

(44) Wriedt, H. A.; Gokcen, N. A.; Nafziger, R. H. Bull. Alloy Phase Diagrams 1987, 8, 355-377.

(45) Petravic, M.; Peter, R.; Fan, L.-J.; Yang, Y.-W.; Chen, Y. Nucl. Instrum. Methods Phys. Res., Sect. A 2010, 619, 94-97.

(46) Liu, Z.; Gong, Y.; Zhou, W.; Ma, L.; Yu, J.; Idrobo, J. C.; Jung, J.; MacDonald, A. H.; Vajtai, R.; Lou, J.; Ajayan, P. M. Nat. Commun. 2013, 4, 2541.

(47) Orlando, F.; Larciprete, R.; Lacovig, P.; Boscarato, I.; Baraldi, A.; Lizzit, S. J. Phys. Chem. C 2012, 116, 157-164.

(48) Müller, F.; Hüfner, S.; Sachdev, H.; Gsell, S.; Schreck, M. Phys. Rev. B: Condens. Matter Mater. Phys. 2010, 82, 1-11.

(49) Li, L.; Li, L. H.; Chen, Y.; Dai, X. J.; Xing, T.; Petravic, M.; Liu, X. Nanoscale Res. Lett. 2012, 7, 417.

(50) Sachdev, H.; Müller, F.; Hüfner, S. Angew. Chem., Int. Ed. 2011, 50, 3701-3705.

(51) Bresnehan, M. S.; Hollander, M. J.; Wetherington, M.; Wang, K.; Miyagi, T.; Pastir, G.; Snyder, D. W.; Gengler, J. J.; Voevodin, A. A.; Mitchel, W. C.; Robinson, J. A. J. Mater. Res. 2014, 29, 459-471.

(52) Trehan, R. J. Vac. Sci. Technol., A 1990, 8, 4026-4032.

(53) Schild, D.; Ulrich, S.; Ye, J.; Stüber, M. Solid State Sci. 2010, 12, 1903-1906.

(54) Cabrero-Vilatela, A.; Weatherup, R. S.; Braeuninger-Weimer, P.; Caneva, S.; Hofmann, S. Nanoscale 2015, DOI: 10.1039/ C5NR06873H.

(55) Rogl, P.; Schuster, J. C. ASM Int. 1992, 32-37.

(56) Weatherup, R. S.; D’Arsié, L.; Cabrero-Vilatela, A.; Caneva, S.; Blume, R.; Robertson, J.; Schlögl, R.; Hofmann, S. J. Am. Chem. Soc. 2015, 137, 14358-14366.

(57) Chou, Y. C.; Wen, C. Y.; Reuter, M. C.; Su, D.; Stach, E. a.; Ross, F. M. ACS Nano 2012, 6, 6407-6415.

(58) Gamalski, A. D.; Perea, D. E.; Yoo, J.; Li, N.; Olszta, M. J.; Colby, R.; Schreiber, D. K.; Ducati, C.; Picraux, S. T.; Hofmann, S. ACS Nano 2013, 7, 7689-7697.

(59) Spizzirri, P. G.; Rubanov, S.; Gauja, E.; Prawer, S. Mater. Forum 2008, 32, 161-166. 